\title{
THE ENVIRONMENT OF HIGH-MASS YOUNG STELLAR OBJECTS
}

\author{
Jean-Pierre Maillard \\ Institut d'Astrophysique de Paris \\ $98 b$ Boulevard Arago \\ Paris 75014 \\ France \\ George F. Mitchell \\ Department of Astronomy \\ Saint Mary's University \\ Halifax, N.S., B3H 3 C3 \\ Canada
}

\begin{abstract}
Using high-resolution infrared $\mathrm{CO}$ spectroscopy new results have been obtained on the environment of high-mass young stellar objects (YSOs). In particular, a new class of neutral, warm and dense outflows has been discovered. The infrared outflows appear a general phenomenon of the activity of high-mass YSOs. With well-defined and often multiple velocities they seem to correspond to episodic and violent mass-loss events from the central source. In addition, a shell of warm, quiescent gas, is formed near the massive star. All these dynamical elements influence the chemistry inside the giant molecular clouds. Beside CO in solid and in gas phase, detection in the infrared has been attempted of simple molecules like $\mathrm{CH}_{4}, \mathrm{C}_{2} \mathrm{H}_{2}, \mathrm{H}_{2} \mathrm{O}$ and ion $\mathrm{H}_{3}^{+}$toward few of these sources.
\end{abstract}

\section{Introduction}

Two types of site for star formation are distinguished, the Giant Molecular Clouds (archetype Orion) and the Dark Cloud Complexes (archetype TaurusAuriga) (Wilking 1989). The latter type forms exclusively low and intermediate mass stars $\left(M_{\star}<2 M_{\odot}\right)$. With high-mass stars $\left(M_{\star} \geq 5 M_{\odot}\right)$ we are dealing with giant clouds in the core of which those stars are grouped in clusters of few units (e.g. S140) or are sometimes single (e.g. M8E-IR). Even if the probability of finding a star with $M_{\star}=5 M_{\odot}$ is 400 times smaller than that of a typical star 
according to current estimates of the Initial Mass Function (Scalo 1990), these massive stars, less numerous, will have a determining impact on the evolution of the giant clouds. They are also the most brightest infrared sources embedded in molecular clouds. From this point of view they present a special interest for probing the gas and dust content of clouds on their line of sight.

This paper will focus on results obtained by infrared high-resolution spectroscopy, in particular from the $\mathrm{CO}$ band at $5 \mu \mathrm{m}$. By detecting lines in absorption, the infrared range does not give access to a chemistry as rich as in the millimetric range. The method presents, however, some specific advantages. The line of sight is observed at high angular resolution $(\leq 1$ "), which avoids the dilution of small scale structures, important to study the close environment of the sources. Very precise information on the kinematics and the column density of the absorbing gas are deduced. Finally, symmetrical molecules not detectable in millimeter such as $\mathrm{CH}_{4}$, are only observable that way. The review extends the pioneering work on $\mathrm{BN}$ to several other high-mass sources, showing various degrees of evolution, to build a picture of the YSOs environment.

\section{Observations}

The CO observations reviewed here were taken using the Fourier Transform Spectrometer at the Cassegrain focus of the Canada-France-Hawaii Telescope on Mauna Kea, mainly between 1987 and 1988. Details of the FTS can be found in Maillard and Michel (1982). The main part of the vibration-rotation band of ${ }^{12} \mathrm{CO}$ and ${ }^{13} \mathrm{CO}(\Delta \mathrm{v}=1)$ was covered, with a spectral resolution of $0.05 \mathrm{~cm}^{-1}$, corresponding at $4.7 \mu \mathrm{m}$ to a velocity resolution of $8 \mathrm{~km} \mathrm{~s}^{-1}$.

\section{Presentation of the sources}

Sources of Bro and $\operatorname{Br} \gamma$ in emission toward molecular clouds (Simon et al. 1983), were the first direct evidence of embedded, hot luminous young stars. The comparison of the intensities of the lines was in addition a way to estimate the amount of dust in the line of sight. The bipolar outflows in millimetric CO (Bally and Lada 1983) centred on YSOs, proved to be another characteristic phenomenon of the first stages of stellar evolution.

The most-studied source toward which the two features are detected is BN. With more CO mappings made, some objects have been found to be source of large scale outflows although the HI recombination lines are not detectable (e.g. GL 2591), and some deeply embedded sources, with infrared luminosity typical of a massive star exhibiting neither a bipolar outflow nor Br $\alpha$ (e.g. W33 A). These observational facts suggest different ages of the central source. W33 A could be considered in the most primitive state, while BN is more evolved, having already created a developed HII region. GL 2591 is in an intermediate situation. At the latest stage, the massive star disrupts its parental cloud and becomes visible. That is $\mathrm{LkH} \alpha$ 101. Table I presents the main parameters of 11 massive sources observed in infrared CO, grouped according to this sequence. Two papers (Mitchell et al. 1990, and Mitchell, Maillard and Hasegawa, 1991) present in more details the results of this study.

\section{Gas and dust properties}

Analysis of the numerous CO line profiles provided by the high-resolution spectra, preferably ${ }^{13} \mathrm{CO}$ to remain in the optically thin approximation, makes it possible to 
Table I: PARAMETERS OF THE YOUNG HIGH-MASS SOURCES

\begin{tabular}{lllllll}
$\begin{array}{l}\text { Source } \\
\text { Name }\end{array}$ & $\begin{array}{l}\text { distance } \\
k p c\end{array}$ & \multicolumn{2}{l}{$\begin{array}{l}\mathrm{L}_{I R} \\
10^{5} \times L_{\odot}\end{array}$} & $\begin{array}{l}\mathrm{B} \alpha \\
10^{-16} W^{-2}\end{array}$ & $\begin{array}{l}\mathrm{B} m_{4.7 \mu m} \\
J y\end{array}$ & $\begin{array}{l}\text { bipolar } \\
\text { outflow }\end{array}$ \\
\hline GL 2136 & 1.7 & 1.8 & no & no & 120 & no \\
W33 A & 3.7 & 0.3 & no & no & 10 & no \\
\hline S140 IRS1 & 0.9 & 0.24 & no & no & 42 & yes \\
GL 2591 & 1.5 & 0.16 & no & no & 240 & yes \\
W3 IRS5 & 2.3 & 4.2 & no & no & 40 & yes \\
NGC 7538 IRS9 & 3.5 & 0.3 & no & no & 28 & yes \\
\hline BN & 0.5 & 1.2 & 194 & 22 & 122 & yes \\
GL 490 & 0.9 & 0.014 & 39 & 9 & 35 & yes \\
M8E-IR & 1.5 & 0.25 & 16 & no & 66 & yes \\
NGC 7538 IRS1 & 3.5 & 1.5 & 50 & no & 40 & yes \\
\hline LkH $\alpha 101$ & 0.8 & 0.14 & 1200 & 420 & 170 & no
\end{tabular}

Table II: RESULTS IN INFRARED CO

\begin{tabular}{lllllll}
$\begin{array}{l}\text { Source } \\
\text { Name }\end{array}$ & $\begin{array}{l}\text { solid } \\
\text { CO }\end{array}$ & $\begin{array}{l}\text { quiescent } \\
{ }^{2} \text { CO }\end{array}$ & $\begin{array}{l}\text { gas } \\
{ }^{3} \text { CO }\end{array}$ & $\begin{array}{l}\text { Infrared } \\
\text { outflows }\end{array}$ & $\begin{array}{l}\text { Nb of } \\
\text { comp. }\end{array}$ & $\begin{array}{l}\text { PCyg } \\
\text { profile }\end{array}$ \\
\hline GL 2136 & $\times$ & $\times$ & $\times$ & no & & \\
W33 A & $\times$ & $\times$ & $\times$ & no & & \\
\hline S140 IRS1 & & $\times$ & $\times$ & yes & 1 & yes \\
GL 2591 & & $\times$ & $\times$ & yes & $>4$ & no \\
W3 IRS5 & $\times$ & $\times$ & $\times$ & yes & 3 & yes \\
NGC 7538 IRS9 & $\times$ & $\times$ & $\times$ & yes & 2 & yes \\
\hline BN & & $\times$ & $\times$ & yes & 3 & yes \\
GL 490 & & $\times$ & & yes & 1 & no \\
M8E-IR & & $\times$ & & yes & $\geq 5$ & no \\
NGC 7538 IRS1 & $\times$ & $\times$ & $\times$ & yes & 1 & yes \\
\hline LkH $\alpha 101$ & & $\times$ & & no & &
\end{tabular}


determine the temperature of the medium in which the lines are formed. Combined with the radial velocity and the density, one can determine the properties of the various absorbing regions in the line of sight. Three regions can be discriminated: the ambient molecular cloud, a quiescent high-density shell, the outflowing gas. The main results are gathered in Table II.

\subsection{Molecular cloud}

The column density in ${ }^{12} \mathrm{CO}$ (assuming a solar ${ }^{12} \mathrm{C} /{ }^{13} \mathrm{C}$ ratio) is between 1 to $2 \times$ $10^{19} \mathrm{~cm}^{-2}$, the density of the gas on the average $10^{5} \mathrm{~cm}^{-3}$. The temperatures deduced from the infrared $\mathrm{CO}$ are in the range 25 to $65 \mathrm{~K}$, consistent with other temperature determinations. The same spectral range gives access also to the absorption band of solid CO at $2140 \mathrm{~cm}^{-1}$. Using solid CO abundances of Sandford et al. (1988) a solid-to-gas phase ratio can be estimated to be $\leq 10 \%$, i.e., most of the $C O$ is in gas phase. These results give typical conditions of giant molecular clouds.

\subsection{Quiescent high-density shell}

On the same lines of sight, the high-J lines are indicative of the presence of a warm quiescent gas, with a temperature different for each source, ranging from 120 to $1000 \mathrm{~K}$, likely heated by gas-grain collisions. The density is higher than in the ambient cloud $\left(\geq 10^{7} \mathrm{~cm}^{-3}\right)$, and the radial velocity can be different by a few $\mathrm{km} \mathrm{s}^{-1}$ toward the blue. By analogy with the spherical VLA images of ultracompact HII regions (Churchwell 1990) that most of these sources are forming, the quiescent gas can be thought to be on a shell where the gas blown away by the central star is piling up. Future high-resolution infrared imaging should be able to confirm this view.

\subsection{Neutral outflowing gas}

That is a general phenomenon we observed for all the sources with developed bipolar outflows. However, this outflowing gas is distinct from the millimetric outflows. The velocity of the gas is high, from 15 to $160 \mathrm{~km} \mathrm{~s}^{-1}$, and the kinetic temperature can reach up to $500 \mathrm{~K}$. Several velocity components are often present, which suggests episodic mass-loss events. Remain to elucidate how they relate to the formation of the large scale sub-millimetric bipolar flows, but their concurrent existence suggests a possible link between the two types of outflow.

\section{Search for other molecules in the infrared}

The existence of a high-density and warm gas shell in the line of sight of the massive YSOs should be favorable to the possible detection in the infrared of neutral molecules in absorption, in gas phase, which locked onto dust are released by warm grains. Search for molecules like $\mathrm{NH}_{3}, \mathrm{OCS}, \mathrm{HCN}, \mathrm{C}_{2} \mathrm{H}_{2}$ in Orion, is reviewed by Evans (1991). Complements for $\mathrm{H}_{2} \mathrm{O}, \mathrm{CH}_{4}$ and $\mathrm{H}_{3}^{+}$are given here.

\section{$5.1 \mathrm{H}_{2} \mathrm{O}$}

The detection of four weak lines of the $\nu_{3}$ band at $2.7 \mu \mathrm{m}$ is reported by Knacke and Larson (1991) toward $\mathrm{BN}$ from the $\mathrm{KAO}$. A ratio $\left[\mathrm{H}_{2} \mathrm{O}\right.$ gas $] /[$ ice $] \leq 0.05$ implies that most of $\mathrm{H}_{2} \mathrm{O}$ is frozen on grains in $\mathrm{OMC}$.

\section{$5.2 \mathrm{CH}_{4}$}

The first positive detection of interstellar gaseous and solid methane has been claimed by Lacy et al. (1991), who have searched for this basic molecule at 7.8 $\mu \mathrm{m}$ toward several of the sources we observed in CO. Another possible region is $3.1 \mu \mathrm{m}$. The observations, because of the telluric methane, are difficult and the most convincing detection is obtained only toward NGC 7538 IRS9. The tentative 
conclusion is that, methane is predominantly in solid phase, with an abundance of solid $\mathrm{CH}_{4}$ comparable to that of solid $\mathrm{CO}$.

\section{$5.3 \mathrm{H}_{3}^{+}$}

The $\mathrm{H}_{3}^{+}$ion is fundamental for the ion-molecule chemistry. Its abundance is also a probe of the cosmic ray ionization in molecular clouds. Several observations have been conducted toward BN, NGC 2024 IRS2, NGC 2264 and GL 2591 (Geballe and Oka 1989, Black et al. 1990) in the $\nu_{2}$ band at $4 \mu \mathrm{m}$ where 6 lines might be usable. No positive detection in any molecular cloud has been reported yet. From new observations toward GL 2591, assuming the canonical value $\mathrm{N}\left[{ }^{12} \mathrm{CO}\right] / \mathrm{N}\left[\mathrm{H}_{2}\right]$ $=10^{-4}$, taking the cold gas temperature of $38 \mathrm{~K}$ and the ${ }^{12} \mathrm{CO}$ column density of $1.1 \times 10^{19} \mathrm{~cm}^{-2}$ (Mitchell et al. 1989), we derived an improved upper limit of

$$
\mathrm{N}\left[\mathrm{H}_{3}^{+}\right] / \mathrm{N}\left[\mathrm{H}_{2}\right]<2.7 \times 10^{-9}
$$

To estimate how this limit is already a test of ion-molecule chemistry, a calculation similar to Herbst and Leung's prediction (1986) made for TMC1 should be conducted for the conditions of a giant molecular cloud.

\section{Conclusion}

By high-resolution absorption spectroscopy of $\mathrm{CO}$, a large number of new results have been collected, which can be extended to more sources to better generalize the conclusions drawn from about ten sources. For the other important interstellar neutral molecules like $\mathrm{H}_{2} \mathrm{O}, \mathrm{CH}_{4}, \mathrm{C}_{2} \mathrm{H}_{2}, \mathrm{NH}_{3}$, and ion $\mathrm{H}_{3}^{+}$, the detection is more difficult, and this research is just beginning. High-mass YSOs are the only infrared sources which give a chance of detecting them. More sensitive high-resolution spectroscopy of these molecules, will provide new insights on the chemical composition of the giant molecular clouds in which massive stars form.

\section{References}

Bally, J. and Lada, C.J., 1983, Ap. J., 265, 824.

Black, J.H, van Dishoeck, E., Willner, S.P., and Woods, R.C., 1990, Ap. J., 358, 459.

Churchwell, E., 1990, Astron. Astrophys. Rev., $2,79$.

Evans, N.J., 1991, this symposium.

Geballe, T.R., and Oka, T., 1989, Ap. J., 342, 855.

Knacke, R.F., and Larson, H.P., 1991, Ap. J., 367, 162.

Herbst, E., and Leung, C.M., 1986, Ap. J., 310, 378.

Lacy, J.H., Carr, J.S., Evans, N.J., Baas, F., Achtermann, J.M., and Arens, J.F., 1991, Ap. J., 376, 556.

Maillard, J.P., and Michel, G., 1982, in Instrumentation for Astronomy with Large Optical telescopes, IAU Col. No. 67, C. M. Humphries ed, Reidel, p. 213.

Mitchell, G.F., Curry, C., Maillard, J.P., and Allen, M. 1989, Ap. J., 341, 1020.

Mitchell, G.F., Maillard, J.P., Allen, M., Beer, R., and Belcourt, K., 1990, Ap. J., 363, 554.

Mitchell, G.F., Maillard, J.P., and Hasegawa,T.I., 1991, Ap. J., 371, 342.

Sandford, S.A., Allamandolla, L.J., Tielens, A.G.G., and Valero, G.J, 1988, Ap. J., $329,498$.

Scalo, J.M., 1990, in Windows on Galaxies, A. Renzini, G. Fabbiano and J. Gallagher eds, Kluwer-Dordrecht, p. 125.

Simon, M., Righini-Cohen, G., Felli, M., and Fischer, J., 1981, Ap. J., $245,552$.

Wilking, B.A., 1989, Pub. A. S. P., 101, 229. 


\section{QUESTIONS AND ANSWERS}

A.Leger: How do you understand $T_{\text {rot }}=10^{3} \mathrm{~K}$ in Jupiter?

J.P.Maillard: That is a completely different problem, not relevant to interestellar medium. I have presented this emission spectrum of $\mathrm{H}_{3}^{+}$on Jupiter just to show what the $\mathrm{H}_{3}^{+}$spectrum looks like and the lines we are looking for in absorption in molecular clouds. The mode of excitation of $\mathrm{H}_{3}^{+}$in Jupiter is totally different. It is an auroral phenomenon.

S.Sandford: (1) What is the central peak position and full-width-at-half-maximum of the solid CO feature in NGC 7538 IRS 9? (2) Are the orientations of the bipolar outflows understood?

J.P.Maillard: (1) This information is in the data. We could look at, but we have been more interested on the abundance of solid $\mathrm{CO}$ to compare to gas phase $\mathrm{CO}$ than do the position and the shape of the band.

(2) There is a systematic study we could conduct, as how the line of sight intersect the large scale flows for instance. We have new maps in ${ }^{12} \mathrm{CO}(2 \rightarrow 1)$ I presented four of these sources which can help. However, the bipolar flows from these massive sources are generally very open, then there is a large probability that the line of sight is within a flow. We have no source so far with a flow like the prototype are for low-mass objects L1551-IRS 5 which is just oriented perpendicular to the line of sight. With such a case we could check if there is an influence on the absorption $\mathrm{CO}$ profile. 